\title{
Relative Frequency of Staphylococcal Carriage and Antibiotic Sensitivity of Isolated Staphylococci in Hemodialysis Patients in Shiraz, Iran
}

\author{
Mohammad Motamedifar $^{\mathrm{a}, \mathrm{b}}$ Parvin Hassanzadeh ${ }^{c}$ Narjes Ghafari ${ }^{\mathrm{d}}$ \\ a Department of Bacteriology and Virology, Shiraz Medical School, bShiraz HIV/AIDS Research Center (SHARC), \\ University of Medical Sciences, 'Biology Department, School of Science, and d Department of Pathobiology, \\ Veterinary School, Shiraz University, Shiraz, Iran
}

\section{Key Words}

Hemodialysis · Staphylococcus aureus · Carriage rate •

Shiraz • Iran

\begin{abstract}
Objective: The purpose of this study was to determine the nasal carriage rate of Staphylococcus aureus in hemodialysis patients and the antibiogram of all isolated $S$. aureus from carriers. Subjects and Methods: A cross-sectional study was carried out from May to September 2007. 130 patients (49 females, 81 males; mean age $55.6 \pm 15.2$ years) with chronic renal failure were included in the study. The patients had undergone hemodialysis for at least 12 months in the dialysis ward of Faghihi Hospital, a referral center in Shiraz, Iran. Swab specimens were taken from the anterior nares and skin of the patients and cultured microbiologically. Next, antibiotic susceptibility tests were performed for all of the isolated S. aureus. Results: 32 (24.6\%) patients (95\% Cl 17.5$32.9 \%)$ were nasal carriers of S. aureus versus $98(75.4 \%)$ non-carriers. S. aureus was also isolated from skin of 5 (3.8\%) patients who were nasal carriers as well. The isolated $S$. aureus were $100,97.3,73.0,70.3,40.5$ and $21.6 \%$ sensitive to vancomycin, rifampin, ciprofloxacin, tetracycline, methicillin and erythromycin, respectively. Conclusion: Because the
\end{abstract}

nasal carriage rate of $S$. aureus observed in dialysis patients in this study was relatively high, we recommend screening for S. aureus in hemodialysis patients to enable prompt prevention strategies.

Copyright $\odot 2010$ S. Karger AG, Basel

\section{Introduction}

Staphylococcus aureus is a habitant bacterium in some sites, such as the throat, skin and anterior nares in humans; however, its carriage can play a key role in the epidemiology and pathogenesis of staphylococcal infections [1]. Studies have shown that the anterior nares are the most consistent site from which this organism can be cultured [2]. Cross-sectional studies yield a prevalence rate of approximately $35 \%$ for $S$. aureus nasal carriage in the general population, which is actually a mix of persistent and intermittent carriers at that time point. Subgroups of patients with significantly increased carriage rates include those with insulin-dependent diabetes mellitus, liver dysfunction, S. aureus skin infections, HIV infection and those on intravenous drugs, chronic ambulatory peritoneal dialysis or hemodialysis $[1,3]$. S. aureus carriage in the nose and on the skin has been shown to be

\section{KARGER}

Fax +4161306 1234

E-Mail karger@karger.ch

www.karger.com
C) 2010 S. Karger AG, Basel

$1011-7571 / 10 / 0195-0379 \$ 26.00 / 0$

Accessible online at:

www.karger.com/mpp
Dr. Mohammad Motamedifar

Department of Bacteriology and Virology

Shiraz Medical School, Shiraz University of Medical Sciences P.O. Box 71455-119, Shiraz 71455 (Iran)

Tel./Fax +98 7112304356 , E-Mail motamedm@sums.ac.ir 
more common in patients receiving chronic hemodialysis than in the general population. A carriage rate of 40.584.0\% has been reported for such patients [4-6].

$S$. aureus is a leading cause of bacteremia among hemodialysis-dependent patients and the rates may be increasing [7]. Persons receiving hemodialysis are especially vulnerable to vascular access infections because they require vascular access for prolonged periods and undergo frequent puncture of their vascular access site. Vascular access is a major risk factor for bacteremia [4, 8], infection-related hospitalization and mortality among hemodialysis patients because of their immunodeficiency [1]. Complications of $S$. aureus bacteremia include sepsis, endocarditis, osteomyelitis, septic arthritis, peritonitis and metastatic abscesses $[9,10]$. On the other hand, hemodialysis patients undergoing long-term hemodialysis, recurrent antimicrobial treatment and immunodeficiency cause increased bacterial resistance and the spread of dangerous hospital infections to other patients. There are many studies [4-6] showing a high incidence rate of staphylococcal carriage in hemodialysis patients, and these carriages are known to be the main risk factor for spreading hospital infections. Therefore, study of the prevalence rate of staphylococcal carriage and drug resistance patterns in staphylococcal isolates can help in determining the rate of infection in order to manage $S$. aureus carriage and prevent serious consequences, including mortality in either hemodialysis patients or others.

Although there are some reports of nasal carriage rates among hospital patients other than those undergoing hemodialysis and also on health care workers in our region $[8,11]$, very little information is available on the prevalence rate of nasal carriage in hemodialysis patients in Iran. Thus, in this cross-sectional study, we aimed to determine the frequency of $S$. aureus nasal carriage in hemodialysis patients in Shiraz, the major city in southwestern Iran, and to investigate the resistance patterns of the isolated $S$. aureus against various antibiotics used broadly for treatment.

\section{Subjects and Methods}

This study was conducted in Faghihi Hospital, a referral dialysis center in Shiraz, Iran. Shiraz is the largest city in the southwest of Iran. Faghihi University Hospital in Shiraz serves as a referral center with more than 10,000 admissions per year.

In a cross-sectional study from May to September 2007, 130 hemodialysis patients who had undergone treatment for at least 12 months were included. Of the 130 patients, 49 were females and
81 were males; mean age was $55.5 \pm 15.2$ years. The patients who had undergone $<12$ months hemodialysis were excluded. Nasal and skin specimens were collected from the hemodialysis patients using 2 cotton-wool swabs for each patient.

Cotton-wool sterile swabs were moistened in normal saline and were rotated in the left and right anterior nares. Skin swabs were obtained from a $3 \times 3 \mathrm{~cm}$ area around the site of access for hemodialysis, before skin preparation. The swabs were immediately inoculated onto sheep blood-agar plates and phenol redmannitol-salt agar (Himedia, India). The plates were incubated at $37^{\circ} \mathrm{C}$ for $48 \mathrm{~h}$ and identification of $S$. aureus was based upon colony morphology, catalase positivity, mannitol fermentation, Gram-positive cocci in clusters on the tube-coagulase test, clumping factor reaction with rabbit plasma on microscope slides, and DNase positivity [12].

The susceptibility of strains to antibacterial agents was determined by using the Kirby-Bauer disc diffusion method (the predominant method employed in Iran), following the guidelines of the Clinical and Laboratory Standards Institute [13]. Each isolate was tested using the following panel of antibiotic discs (Mast Diagnostics, UK) including vancomycin $(30 \mu \mathrm{g})$, rifampin $(5 \mu \mathrm{g})$, ciprofloxacin $(5 \mu \mathrm{g})$, tetracycline $(10 \mu \mathrm{g})$, methicillin $(5 \mu \mathrm{g})$ and erythromycin $(15 \mu \mathrm{g})$. The standard sensitive strain of $S$. aureus ATCC 25923 was included as a control for reproducibility of results with every batch of antibiotic sensitivity test.

Owing to the high risk of staphylococcal infections in hemodialysis patients, those isolates showing intermediate susceptibility to an antimicrobial agent were categorized as resistant isolates for data analysis and presentation.

The results were statistically analyzed and the groups were compared by $\chi^{2}$ tests using SPSS, version 11.5. Differences between groups were considered statistically significant if $\mathrm{p}<0.05$.

\section{Results}

$32(24.6 \%)$ patients were identified as $S$. aureus nasal carriers (95\% CI 17.5-32.9\%). There was no significant difference between male and female nasal carriers. The lowest and the highest prevalence rates of nasal carriage were observed in the 20 - to 30 -year and the 71 - to 80 -year age groups, respectively, and the difference was statistically significant $(\mathrm{p}<0.05)$. Age and gender distributions of $S$. aureus nasal carriers are presented in table 1. S. aureus was also isolated from skin of $5(3.8 \%)$ patients who were nasal carrier as well: 3 males and 2 females.

Based on clinical reports, among the 32 nasal carriers; $15(53.6 \%)$ had a past history of hypertension, 4 (14.3\%) of insulin-dependent diabetes mellitus, 3 (10.7\%) of both diabetes and hypertension, and $6(21.2 \%)$ of renal dysfunctions. No significant association was detected between $S$. aureus nasal carriage and having a past history of hypertension, diabetes mellitus or renal dysfunctions. 
The prevalence of antibacterial sensitivities in 37 isolated S. aureus is given in table 2 . The highest susceptibility results were observed against vancomycin, rifampin and ciprofloxacin, and the lowest ones were against methicillin and erythromycin.

\section{Discussion}

S. aureus, a frequent cause of infections in both the community and hospital, is the most common organism responsible for intravenous catheter-related infection, postoperative wound infection, septicemia, septic arthritis, and skin and soft tissue endogenous infections in hemodialysis patients $[7,9,10,14,15]$. A major part of dialysis-related infection is endogenous and related to the high frequency of nasal and skin carriage of S. aureus [16]. The nose is the main ecological niche where $S$. aureus resides in human beings, but the determinants of the carrier state are incompletely understood. It has been shown that nasal carriers of $S$. aureus have an increased risk of acquiring an infection with this pathogen. Eradication of $S$. aureus from nasal carriers prevents infection in specific patient categories, including hemodialysis patients [17]. Worldwide, the increasing resistance of this pathogen to various antibiotics complicates treatment of $S$. aureus infections. Therefore, effective measures to prevent $S$. aureus infections are urgently needed.

In the present study, a mean $S$. aureus carriage rate of $24.6 \%$ was observed. However, this rate is lower than those reported in other studies (40.5-84.0\%) [4-6]. The difference between the lower prevalence rate observed in our study and those of other studies may be partly due to differences in the quality of sampling and to the culture techniques used in different studies. For example, in a study in northeastern Iran with a smaller sample, $40.5 \%$ of 69 hemodialysis patients were reported to have $S$. aureus in their nose [6]. Some studies reported that when patients were hospitalized, insulin-dependent diabetes mellitus, hemodialysis treatment, intravenous drug addiction, $S$. aureus skin infections and HIV infection increased carriage rates significantly $[1,3]$.

The higher prevalence rate in older patients ( $>71$ years) in our study confirmed the finding that older age was associated with the risk of $S$. aureus nasal carriage [16-18]. Further, Saxena and Panhotra [16] reported that patients $>65$ years had a significantly higher prevalence of nasal carriage of $S$. aureus and carried an approximately 12.5 times greater combined risk of developing S. aureus- and vascular access-related septicemia than the youngest age
Table 1. Age distribution and prevalence of $S$. aureus nasal carriage in 130 patients under study

\begin{tabular}{llll}
\hline & $\begin{array}{l}\text { S. aureus } \\
\text { carriers }\end{array}$ & $\begin{array}{l}\text { S. aureus } \\
\text { non-carriers }\end{array}$ & Total \\
\hline $\begin{array}{l}\text { Female } \\
\text { Male }\end{array}$ & $13(26.5 \%)$ & $36(73.5 \%)$ & $49(37.7 \%)$ \\
$\begin{array}{l}\text { Age group } \\
\text { 20-30 }\end{array}$ & $19(23.5 \%)$ & $62(76.5 \%)$ & $81(62.3 \%)$ \\
$31-40$ & $3(23.1 \%)$ & $12(100 \%)$ & $12(9.2 \%)$ \\
$41-50$ & $5(26.3 \%)$ & $14(73.7 \%)$ & $13(10 \%)$ \\
$51-60$ & $7(23.3 \%)$ & $23(76.7 \%)$ & $30(23.1 \%)$ \\
$61-70$ & $7(21.9 \%)$ & $25(78.1 \%)$ & $32(24.7 \%)$ \\
$71-80$ & $9(40.9 \%)$ & $13(59.1 \%)$ & $22(16.9 \%)$ \\
$81-90$ & $1(50.0 \%)$ & $1(50 \%)$ & $2(1.5 \%)$ \\
\hline Total & 32 & 98 & $130(100 \%)$ \\
\hline
\end{tabular}

Table 2. Prevalence of antibacterial sensitivities in 37 isolated S. aureus

\begin{tabular}{lr}
\hline & Sensitivity \\
\hline Ciprofloxacin & $27(73.0 \%)$ \\
Erythromycin & $8(21.6 \%)$ \\
Methicillin & $15(40.5 \%)$ \\
Rifampin & $36(97.3 \%)$ \\
Vancomycin & $37(100 \%)$ \\
Tetracycline & $26(70.3 \%)$ \\
\hline
\end{tabular}

group. These findings might be helpful in the identification of elderly hemodialysis patients as a high-risk group for $S$. aureus-linked vascular access-related septicemia and to develop appropriate preventive strategies.

In our study, male patients had a higher prevalence of $S$. aureus nasal carriage, but no significant difference was detected between male and female subjects. Similarly, gender was not considered as an important risk factor for $S$. aureus carriers in hemodialysis patients in another study [19].

No significant association was detected between $S$. aureus nasal carriage and having a past history of hypertension, diabetes mellitus or renal dysfunctions. Similarly, a study reported that diabetes mellitus was not a significant risk factor for nasal carriage [19]. However, there are some researchers reporting insulin-dependent diabetes mellitus as an increasing factor for $S$. aureus carriage rates $[1,3]$. 
Several oral and topical antibiotics for eradication of S. aureus nasal carriage in hemodialysis patients have been studied [8]. Similar to some other studies [20,21], we found high levels of resistance to tetracycline, methicillin and erythromycin. In our study, more than half of the $S$. aureus isolates were methicillin-resistant $S$. aureus (MRSA). In a study in another hospital of Shiraz, MRSA nasal carriage rate was reported to be $42.4 \%$ among hospital patients [11], which is more than that observed in our study. However, one study has shown that the rates of MRSA isolates have increased steadily over the past decade [15]. Most of our isolates were sensitive to vancomycin, rifampin and ciprofloxacin. Rifampin has been shown to be the most effective oral agent used for S. aureus eradication [4]. Kluytmans et al. [17] showed that mupirocin nasal ointment effectively eliminated nasal carriage of $S$. aureus in patients on hemodialysis, but a steadily increasing resistance to mupirocin has been reported in Kuwaiti hospitals in recent years [20].

Smith et al. [22] reported that vancomycin was the only uniformly effective treatment for staphylococcal infections, although reduced susceptibility to vancomycin has also recently been reported [15].

In this study, carrier status was based only on a single culture, which does not differentiate permanent carriers from intermittent carriers. However, there is increasing evidence showing that higher numbers of staphylococci constitute a higher risk of infections by the patients' own strain in permanent carriers.
In our study the nasal carriage rates of $S$. aureus in dialysis patients were less than in some other studies [4-6]; however, as even a low rate of staphylococcal nasal carriage is of significance, precise screening of the $S$. aureus carrier state in hemodialysis patients, including the antibiotic sensitivity test if possible, is vital to adequately target prevention strategies (such as using nasal ointments). Other measures of prevention (e.g. vaccines for staphylococcal infections) can be considered, as a conjugate vaccine in hemodialysis patients has been shown to confer partial immunity against $S$. aureus bacteremia for approximately 40 weeks, after which protection wanes as antibody levels decrease [23].

\section{Conclusion}

The nasal carriage rate of $S$. aureus observed in dialysis patients in this study was relatively high, so screening of the $S$. aureus carrier state in our hemodialysis patients for prompt prevention strategies is recommended. No vancomycin-resistant $S$. aureus was observed in our study. However, continued determination of the emergence of glycopeptide-resistant isolates of $S$. aureus remains a prudent strategy.

\section{Acknowledgements}

This work was financially supported by a Shiraz University research grant (No. 86-SC-1806-C355) and a Shiraz University of Medical Sciences research grant (No. 86-3413).

\section{References}

1 Kluytmans J, van Belkum A, Verbrugh H: Nasal carriage of Staphylococcus aureus: epidemiology, undergoing mechanisms, and associated risks. Clin Microbiol Rev 1997;10: 505-520.

-2 Solberg CO: Spread of Staphylococcus aureus in hospitals: causes and prevention. Scand J Infect Dis 2000;32:587-595.

- 3 Nouwen JL, van Belkum A, Verbrugh HA Determination of Staphylococcus aureus nasal carriage. Neth J Med 2001;59:126-133.

- 4 Yu VL, Goetz A, Wagener M, Smith PB, Rihs JD, Hanchett J, Zuravleff JJ: Staphylococcus aureus nasal carriage and infections in patients on hemodialysis: efficacy of antibiotic prophylaxis. N Engl J Med 1986;315:91-96.
5 Kaplowitz LG, Comstock JA, Landwehr DM, Dalton HP, Mayhall CG: Prospective study of microbial colonization of the nose and skin and infection of the vascular access site in hemodialysis patients. J Clin Microbiol 1988;26:1257-1262.

6 Ghazvini K, Hekmat R: Nasal and skin colonization of Staphylococcus aureus in hemodialysis patients in northeast of Iran. Iran J Kidney Dis 2007;1:21-24.

-7 Marr KA, Sexton DJ, Conlon PJ, Corey GR, Schwab SJ, Kirkland KB: Catheter-related bacteremia and outcome of attempted salvage in patients undergoing hemodialysis. Ann Intern Med 1997;127:275-280.

-8 Powe NR, Jaar B, Furth SL, Hermann J, Briggs W: Septicemia in dialysis patients: incidence, risk factors, and prognosis. Kidney Int 1999;55:1081-1090.
-9 Marr KA, Kong L, Fowler VG, Gopal A, Sexton DJ, Conlon PJ, Corey GR: Incidence and outcome of Staphylococcus aureus bacteremia in hemodialysis patients. Kidney Int 1998;54:1684-1689.

10 Sexton DJ: Vascular access infections in patients undergoing hemodialysis with special emphasis on the treatment of Staphylococcus aureus. Infect Dis Clin North Am 2000;15: 731-742.

11 Askarian M, Zeinalzadeh A, Japoni A, Alborzi A, Memish ZA: Prevalence of nasal carriage of methicillin-resistant Staphylococcus aureus and its antibiotic susceptibility pattern in healthcare workers at Namazi hospital, Shiraz, Iran. Int J Infect Dis 2009; 13:e241-e247. 
12 Holt JG, Krieg NR, Sneath PHA, Staley JT, Williams ST: Bergey's Manual of Determinative Bacteriology, ed 9. Baltimore, William and Wilkins, 1994, p 532.

13 Clinical and Laboratory Standards Institute: Performance Standards for Antimicrobial Susceptibility Testing (sixteenth informational supplement M100-S16). Wayne, CLSI, 2006.

14 Lowy FD: Staphylococcus aureus infections. N Engl J Med 1998;339:520-532.

15 Udo EE, Al-Sweih N, Dhar R, Dimitrov TS Mokaddas EM, Johny M, Al-Obaid IA, Gomaa HH, Mobasher LA, Rotimi A, Al-Asar A: Surveillance of antibacterial resistance in Staphylococcus aureus isolated in Kuwaiti hospitals. Med Princ Pract 2008;17:71-75.
6 Saxena AK, Panhotra BR: The prevalence of nasal carriage of Staphylococcus aureus and associated vascular access-related septicemia among patients on hemodialysis in $\mathrm{Al}$ Hasa region of Saudi Arabia. Saudi J Kidney Dis Transpl 2003;14:30-38.

17 Kluytmans JW, Manders MJ, van Bommel E, Verbrugh $\mathrm{H}$ : Elimination of nasal carriage of Staphylococcus aureus in hemodialysis patients. Infect Control Hosp Epidemiol 1996; 17:793-797.

18 Saxena AK, Panhotra BR, Chopra R: Advancing age and the risk of nasal carriage of Staphylococcus aureus among patients on long-term hospital-based hemodialysis. Ann Saudi Med 2004;24:337-342.

19 Duran N, Ocak S, Eskiocak AF: Staphylococcus aureus nasal carriage among the diabetic and non-diabetic haemodialysis patients. Int J Clin Pract 2006;60:1204-1209.

20 Udo EE, Al-Sweih N, Mohanakrishnan S, West PWJ: Antibacterial resistance and molecular typing of methicillin-resistant Staphylococcus aureus in a Kuwaiti general hospital. Med Princ Pract 2006;15:39-45.
Udo EE, Panigrahi D, Jamsheer AE: Molecular typing of methicillin-resistant Staphylococcus aureus isolated in a Bahrain hospital. Med Princ Pract 2008;17:308-314.

22 Smith TL, Pearson ML, Wilcox KR, Cruz C, Lancaster MV, Robinson-Dunn B, Tenover FC, Zervos MJ, Band JD, White E, Jarvis WR: Emergence of vancomycin resistance in Staphylococcus aureus. Glycopeptide-Intermediate Staphylococcus aureus Working Group. N Engl J Med 1999;340:493-501.

23 Shinefield H, Black S, Fattom A, Horwith G, Rasgon S, Ordonez J, Yeoh H, Law D, Robbins JB, Schneerson R, Muenz L, Fuller S, Johnson J, Fireman B, Alcorn H, Naso R: Use of a Staphylococcus aureus conjugate vaccine in patients receiving hemodialysis. $\mathrm{N}$ Engl J Med 2002;346:491-496. 